\title{
Multilinear Singular and Fractional Integral Operators on Weighted Morrey Spaces
}

\author{
Hua Wang ${ }^{1}$ and Wentan Yi ${ }^{2}$ \\ ${ }^{1}$ College of Mathematics and Econometrics, Hunan University, Changsha 410082, China \\ ${ }^{2}$ Department of Applied Mathematics, Zhengzhou Information Science and Technology Institute, Zhengzhou 450002, China
}

Correspondence should be addressed to Hua Wang; wanghua@pku.edu.cn

Received 2 May 2013; Accepted 12 September 2013

Academic Editor: John R. Akeroyd

Copyright (c) $2013 \mathrm{H}$. Wang and W. Yi. This is an open access article distributed under the Creative Commons Attribution License, which permits unrestricted use, distribution, and reproduction in any medium, provided the original work is properly cited.

We will study the boundedness properties of multilinear Calderón-Zygmund operators and multilinear fractional integrals on products of weighted Morrey spaces with multiple weights.

\section{Introduction and Main Results}

Multilinear Calderón-Zygmund theory is a natural generalization of the linear case. The initial work on the class of multilinear Calderón-Zygmund operators was done by Coifman and Meyer in [1] and was later systematically studied by Grafakos and Torres in [2-4]. Let $\mathbb{R}^{n}$ be the $n$-dimensional Euclidean space, and let $\left(\mathbb{R}^{n}\right)^{m}=\mathbb{R}^{n} \times \cdots \times \mathbb{R}^{n}$ be the $m$-fold product space $(m \in \mathbb{N})$. We denote by $\mathcal{S}\left(\mathbb{R}^{n}\right)$ the space of all Schwartz functions on $\mathbb{R}^{n}$ and by $\mathcal{S}^{\prime}\left(\mathbb{R}^{n}\right)$ its dual space, the set of all tempered distributions on $\mathbb{R}^{n}$. Let $m \geq 2$ and $T$ be an $m$-linear operator initially defined on the $m$-fold product of Schwartz spaces, and taking values into the space of tempered distributions,

$$
T: \mathcal{S}\left(\mathbb{R}^{n}\right) \times \cdots \times \mathcal{S}\left(\mathbb{R}^{n}\right) \longrightarrow \mathcal{S}^{\prime}\left(\mathbb{R}^{n}\right) .
$$

Following [2], for given $\vec{f}=\left(f_{1}, \ldots, f_{m}\right)$, we say that $T$ is an $m$-linear Calderón-Zygmund operator if, for some $q_{1}, \ldots, q_{m} \in[1, \infty)$ and $q \in(0, \infty)$ with $1 / q=\sum_{k=1}^{m} 1 / q_{k}$, it extends to a bounded multilinear operator from $L^{q_{1}}\left(\mathbb{R}^{n}\right) \times$ $\cdots \times L^{q_{m}}\left(\mathbb{R}^{n}\right)$ into $L^{q}\left(\mathbb{R}^{n}\right)$ and if there exists a kernel function $K\left(x, y_{1}, \ldots, y_{m}\right)$ in the class $m-C Z K(A, \varepsilon)$, defined away from the diagonal $x=y_{1}=\cdots=y_{m}$ in $\left(\mathbb{R}^{n}\right)^{m+1}$ such that

$$
\begin{aligned}
T & (\vec{f})(x) \\
& =T\left(f_{1}, \ldots, f_{m}\right)(x) \\
& =\int_{\left(\mathbb{R}^{n}\right)^{m}} K\left(x, y_{1}, \ldots, y_{m}\right) f_{1}\left(y_{1}\right) \cdots f_{m}\left(y_{m}\right) d y_{1} \cdots d y_{m},
\end{aligned}
$$

whenever $f_{1}, \ldots, f_{m} \in \mathcal{S}\left(\mathbb{R}^{n}\right)$ and $x \notin \bigcap_{k=1}^{m}$ supp $f_{k}$. We say that $K\left(x, y_{1}, \ldots, y_{m}\right)$ is a kernel in the class $m-C Z K(A, \varepsilon)$ if it satisfies the size condition

$$
\left|K\left(x, y_{1}, \ldots, y_{m}\right)\right| \leq \frac{A}{\left(\left|x-y_{1}\right|+\cdots+\left|x-y_{m}\right|\right)^{m n}}
$$

for some $A>0$ and all $\left(x, y_{1}, \ldots, y_{m}\right) \in\left(\mathbb{R}^{n}\right)^{m+1}$ with $x \neq y_{k}$ for some $1 \leq k \leq m$. Moreover, for some $\varepsilon>0$, it satisfies the regularity condition that

$$
\begin{gathered}
\left|K\left(x, y_{1}, \ldots, y_{m}\right)-K\left(x^{\prime}, y_{1}, \ldots, y_{m}\right)\right| \\
\leq \frac{A \cdot\left|x-x^{\prime}\right|^{\varepsilon}}{\left(\left|x-y_{1}\right|+\cdots+\left|x-y_{m}\right|\right)^{m n+\varepsilon}}
\end{gathered}
$$

whenever $\left|x-x^{\prime}\right| \leq(1 / 2) \max _{1 \leq k \leq m}\left|x-y_{k}\right|$ and also that, for each fixed $k$ with $1 \leq k \leq m$,

$$
\begin{aligned}
& \left|K\left(x, y_{1}, \ldots, y_{k}, \ldots, y_{m}\right)-K\left(x, y_{1}, \ldots, y_{k}^{\prime}, \ldots, y_{m}\right)\right| \\
& \quad \leq \frac{A \cdot\left|y_{k}-y_{k}^{\prime}\right|^{\varepsilon}}{\left(\left|x-y_{1}\right|+\cdots+\left|x-y_{m}\right|\right)^{m n+\varepsilon}}
\end{aligned}
$$

whenever $\left|y_{k}-y_{k}^{\prime}\right| \leq(1 / 2) \max _{1 \leq i \leq m}\left|x-y_{i}\right|$. In recent years, many authors have been interested in studying the boundedness of these operators on function spaces; see, for example, [5-8]. In 2009, the weighted strong and weak type estimates of multilinear Calderón-Zygmund singular integral operators were established in [9] by Lerner et al. New more 
refined multilinear maximal function was defined and used in [9] to characterize the class of multiple $A_{\vec{P}}$ weights.

Theorem A (see [9]). Let $m \geq 2$ and $T$ be an $m$-linear Calderón-Zygmund operator. If $p_{1}, \ldots, p_{m} \in(1, \infty)$ and $p \in(0, \infty)$ with $1 / p=\sum_{k=1}^{m} 1 / p_{k}$ and $\vec{w}=\left(w_{1}, \ldots, w_{m}\right)$ satisfies the $A_{\vec{P}}$ condition, then there exists a constant $C>0$ independent of $\vec{f}=\left(f_{1}, \ldots, f_{m}\right)$ such that

$$
\|T(\vec{f})\|_{L^{p}\left(v_{\vec{w}}\right)} \leq C \prod_{i=1}^{m}\left\|f_{i}\right\|_{L^{p_{i}}\left(w_{i}\right)},
$$

where $\nu_{\vec{w}}=\prod_{i=1}^{m} w_{i}^{p / p_{i}}$.

Theorem B (see [9]). Let $m \geq 2$, and let $T$ be an $m$ linear Calderón-Zygmund operator. If $p_{1}, \ldots, p_{m} \in[1, \infty)$, $\min \left\{p_{1}, \ldots, p_{m}\right\}=1$ and $p \in(0, \infty)$ with $1 / p=\sum_{k=1}^{m} 1 / p_{k}$, and $\vec{w}=\left(w_{1}, \ldots, w_{m}\right)$ satisfies the $A_{\vec{p}}$ condition, then there exists a constant $C>0$ independent of $\vec{f}=\left(f_{1}, \ldots, f_{m}\right)$ such that

$$
\|T(\vec{f})\|_{W L^{p}\left(v_{\vec{w}}\right)} \leq C \prod_{i=1}^{m}\left\|f_{i}\right\|_{L^{p_{i}}\left(w_{i}\right)},
$$

where $\nu_{\vec{w}}=\prod_{i=1}^{m} w_{i}^{p / p_{i}}$.

Let $m \geq 2$, and let $0<\alpha<m n$. For given $\vec{f}=\left(f_{1}, \ldots, f_{m}\right)$, the $m$-linear fractional integral operator is defined by

$$
\begin{aligned}
I_{\alpha}(\vec{f})(x) & =I_{\alpha}\left(f_{1}, \ldots, f_{m}\right)(x) \\
& =\int_{\left(\mathbb{R}^{n}\right)^{m}} \frac{f_{1}\left(y_{1}\right) \cdots f_{m}\left(y_{m}\right)}{\left|\left(x-y_{1}, \ldots, x-y_{m}\right)\right|^{m n-\alpha}} d y_{1} \cdots d y_{m} .
\end{aligned}
$$

For the boundedness properties of multilinear fractional integrals on various function spaces, we refer the reader to [10-16]. In 2009, Moen [17] considered the weighted norm inequalities for multilinear fractional integral operators and constructed the class of multiple $A_{\vec{P}, q}$ weights (see also [18]).

Theorem C (see $[17,18])$. Let $m \geq 2,0<\alpha<m n$, and let $I_{\alpha}$ be an $m$-linear fractional integral operator. If $p_{1}, \ldots, p_{m} \in(1, \infty)$, $1 / p=\sum_{k=1}^{m} 1 / p_{k}$ and $1 / q=1 / p-\alpha / n$, and $\vec{w}=\left(w_{1}, \ldots, w_{m}\right)$ satisfies the $A_{\vec{P}, q}$ condition, then there exists a constant $C>0$ independent of $\vec{f}=\left(f_{1}, \ldots, f_{m}\right)$ such that

$$
\left\|I_{\alpha}(\vec{f})\right\|_{L^{q}\left(\left(v_{\vec{w}}\right)^{q}\right)} \leq C \prod_{i=1}^{m}\left\|f_{i}\right\|_{L^{p_{i}}\left(w_{i}^{p_{i}}\right)}
$$

where $\nu_{\vec{w}}=\prod_{i=1}^{m} w_{i}$.

Theorem D (see $[17,18])$. Let $m \geq 2,0<\alpha<m n$, and let $I_{\alpha}$ be an $m$-linear fractional integral operator. If $p_{1}, \ldots, p_{m} \in[1, \infty)$, $\min \left\{p_{1}, \ldots, p_{m}\right\}=1,1 / p=\sum_{k=1}^{m} 1 / p_{k}$ and $1 / q=1 / p-\alpha / n$, and $\vec{w}=\left(w_{1}, \ldots, w_{m}\right)$ satisfies the $A_{\vec{P}, q}$ condition, then there exists a constant $C>0$ independent of $\vec{f}=\left(f_{1}, \ldots, f_{m}\right)$ such that

$$
\left\|I_{\alpha}(\vec{f})\right\|_{W L^{q}\left(\left(v_{\vec{w}}\right)^{q}\right)} \leq C \prod_{i=1}^{m}\left\|f_{i}\right\|_{L^{p_{i}}\left(w_{i}^{p_{i}}\right)},
$$

where $v_{\vec{w}}=\prod_{i=1}^{m} w_{i}$.

On the other hand, the classical Morrey spaces $\mathscr{L}^{p, \lambda}$ were originally introduced by Morrey in [19] to study the local behavior of solutions to second-order elliptic partial differential equations. For the boundedness of the HardyLittlewood maximal operator, the fractional integral operator, and the Calderón-Zygmund singular integral operator on these spaces, we refer the reader to [20-22]. For the properties and applications of classical Morrey spaces, one can see [23$25]$ and the references therein.

In 2009, Komori and Shirai [26] first defined the weighted Morrey spaces $L^{p, \kappa}(w)$ which could be viewed as an extension of weighted Lebesgue spaces and studied the boundedness of the above classical operators in Harmonic Analysis on these weighted spaces. Recently, in [27-34], we have established the continuity properties of some other operators and their commutators on the weighted Morrey spaces $L^{p, \kappa}(w)$.

The main purpose of this paper is to establish the boundedness properties of multilinear Calderón-Zygmund operators and multilinear fractional integrals on products of weighted Morrey spaces with multiple weights. We now formulate our main results as follows.

Theorem 1. Let $m \geq 2$, and let $T$ be an $m$-linear CalderónZygmund operator. If $p_{1}, \ldots, p_{m} \in(1, \infty)$ and $p \in(0, \infty)$ with $1 / p=\sum_{k=1}^{m} 1 / p_{k}$ and $\vec{w}=\left(w_{1}, \ldots, w_{m}\right) \in A_{\vec{p}}$ with $w_{1}, \ldots, w_{m} \in A_{\infty}$, then for any $0<\kappa<1$, there exists $a$ constant $C>0$ independent of $\vec{f}=\left(f_{1}, \ldots, f_{m}\right)$ such that

$$
\|T(\vec{f})\|_{L^{p, \kappa}\left(v_{\vec{w}}\right)} \leq C \prod_{i=1}^{m}\left\|f_{i}\right\|_{L^{p_{i}, \kappa}\left(w_{i}\right)},
$$

where $\nu_{\vec{w}}=\prod_{i=1}^{m} w_{i}^{p / p_{i}}$.

Theorem 2. Let $m \geq 2$ and $T$ be an m-linear CalderónZygmund operator. If $p_{1}, \ldots, p_{m} \in[1, \infty), \min \left\{p_{1}, \ldots, p_{m}\right\}=$ 1 and $p \in(0, \infty)$ with $1 / p=\sum_{k=1}^{m} 1 / p_{k}$, and $\vec{w}=$ $\left(w_{1}, \ldots, w_{m}\right) \in A_{\vec{P}}$ with $w_{1}, \ldots, w_{m} \in A_{\infty}$, then for any $0<\kappa<1$, there exists a constant $C>0$ independent of $\vec{f}=\left(f_{1}, \ldots, f_{m}\right)$ such that

$$
\|T(\vec{f})\|_{W L^{p, \kappa}\left(v_{\vec{w}}\right)} \leq C \prod_{i=1}^{m}\left\|f_{i}\right\|_{L^{p_{i}, \kappa}\left(w_{i}\right)}
$$

where $\nu_{\vec{w}}=\prod_{i=1}^{m} w_{i}^{p / p_{i}}$.

Theorem 3. Let $m \geq 2$, let $0<\alpha<m n$, and let $I_{\alpha}$ be an m-linear fractional integral operator. If $p_{1}, \ldots, p_{m} \in$ $(1, \infty), 1 / p=\sum_{k=1}^{m} 1 / p_{k}, 1 / q_{k}=1 / p_{k}-\alpha / m n$ and $1 / q=$ $\sum_{k=1}^{m} 1 / q_{k}=1 / p-\alpha / n$, and $\vec{w}=\left(w_{1}, \ldots, w_{m}\right) \in A_{\vec{P}, q}$ with 
$w_{1}^{q_{1}}, \ldots, w_{m}^{q_{m}} \in A_{\infty}$, then for any $0<\kappa<p / q$, there exists a constant $C>0$ independent of $\vec{f}=\left(f_{1}, \ldots, f_{m}\right)$ such that

$$
\left\|I_{\alpha}(\vec{f})\right\|_{L^{q, \kappa q / p}\left(\left(v_{\vec{w}}\right)^{q}\right)} \leq C \prod_{i=1}^{m}\left\|f_{i}\right\|_{L^{p_{i} \kappa p_{i} q / p q_{i}}\left(w_{i}^{p_{i}}, w_{i}^{q_{i}}\right)}
$$

where $\nu_{\vec{w}}=\prod_{i=1}^{m} w_{i}$.

Theorem 4. Let $m \geq 2$, let $0<\alpha<m n$, and let $I_{\alpha}$ be an $m$-linear fractional integral operator. If $p_{1}, \ldots, p_{m} \in[1, \infty)$, $\min \left\{p_{1}, \ldots, p_{m}\right\}=1,1 / p=\sum_{k=1}^{m} 1 / p_{k}, 1 / q_{k}=1 / p_{k}-\alpha / m n$ and $1 / q=\sum_{k=1}^{m} 1 / q_{k}=1 / p-\alpha / n$, and $\vec{w}=\left(w_{1}, \ldots, w_{m}\right) \epsilon$ $A_{\vec{P}, q}$ with $w_{1}^{q_{1}}, \ldots, w_{m}^{q_{m}} \in A_{\infty}$, then, for any $0<\kappa<p / q$, there exists a constant $C>0$ independent of $\vec{f}=\left(f_{1}, \ldots, f_{m}\right)$ such that

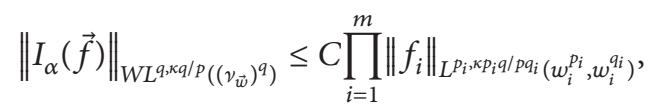

where $v_{\vec{w}}=\prod_{i=1}^{m} w_{i}$.

\section{Notations and Definitions}

The classical $A_{p}$ weight theory was first introduced by Muckenhoupt in the study of weighted $L^{p}$ boundedness of Hardy-Littlewood maximal functions in [35]. A weight $w$ is a nonnegative, locally integrable function on $\mathbb{R}^{n} ; B=B\left(x_{0}, r_{B}\right)$ denotes the ball with the center $x_{0}$ and radius $r_{B}$. For $1<p<$ $\infty$, a weight function $w$ is said to belong to $A_{p}$ if there is a constant $C>0$ such that, for every ball $B \subseteq \mathbb{R}^{n}$,

$$
\left(\frac{1}{|B|} \int_{B} w(x) d x\right)\left(\frac{1}{|B|} \int_{B} w(x)^{-1 /(p-1)} d x\right)^{p-1} \leq C,
$$

where $|B|$ denotes the Lebesgue measure of $B$. For the case $p=1, w \in A_{1}$, if there is a constant $C>0$ such that for every ball $B \subseteq \mathbb{R}^{n}$,

$$
\frac{1}{|B|} \int_{B} w(x) d x \leq C \cdot \underset{x \in B}{\operatorname{ess} \inf } w(x)
$$

A weight function $w \in A_{\infty}$ if it satisfies the $A_{p}$ condition for some $1<p<\infty$. We also need another weight class $A_{p, q}$ introduced by Muckenhoupt and Wheeden in [36]. A weight function $w$ belongs to $A_{p, q}$ for $1<p<q<\infty$ if there is a constant $C>0$ such that, for every ball $B \subseteq \mathbb{R}^{n}$,

$$
\left(\frac{1}{|B|} \int_{B} w(x)^{q} d x\right)^{1 / q}\left(\frac{1}{|B|} \int_{B} w(x)^{-p^{\prime}} d x\right)^{1 / p^{\prime}} \leq C .
$$

When $p=1, w$ is in the class $A_{1, q}$ with $1<q<\infty$ if there is a constant $C>0$ such that, for every ball $B \subseteq \mathbb{R}^{n}$,

$$
\left(\frac{1}{|B|} \int_{B} w(x)^{q} d x\right)^{1 / q}\left(\underset{x \in B}{\operatorname{ess} \sup _{x}} \frac{1}{w(x)}\right) \leq C .
$$

Now let us recall the definitions of multiple weights. For $m$ exponents $p_{1}, \ldots, p_{m}$, we will write $\vec{P}$ for the vector
$\vec{P}=\left(p_{1}, \ldots, p_{m}\right)$. Let $p_{1}, \ldots, p_{m} \in[1, \infty)$, and let $p \in$ $(0, \infty)$ with $1 / p=\sum_{k=1}^{m} 1 / p_{k}$. Given $\vec{w}=\left(w_{1}, \ldots, w_{m}\right)$, set $\nu_{\vec{w}}=\prod_{i=1}^{m} w_{i}^{p / p_{i}}$. We say that $\vec{w}$ satisfies the $A_{\vec{P}}$ condition if it satisfies

$$
\sup _{B}\left(\frac{1}{|B|} \int_{B} \nu_{\vec{w}}(x) d x\right)^{1 / p} \prod_{i=1}^{m}\left(\frac{1}{|B|} \int_{B} w_{i}(x)^{1-p_{i}^{\prime}} d x\right)^{1 / p_{i}^{\prime}}<\infty
$$

When $p_{i}=1,\left((1 /|B|) \int_{B} w_{i}(x)^{1-p_{i}^{\prime}} d x\right)^{1 / p_{i}^{\prime}}$ is understood as $\left(\inf _{x \in B} w_{i}(x)\right)^{-1}$.

Let $p_{1}, \ldots, p_{m} \in[1, \infty)$, let $1 / p=\sum_{k=1}^{m} 1 / p_{k}$, and let $q>$ 0 . Given $\vec{w}=\left(w_{1}, \ldots, w_{m}\right)$, set $\nu_{\vec{w}}=\prod_{i=1}^{m} w_{i}$. We say that $\vec{w}$ satisfies the $A_{\vec{P}, q}$ condition if it satisfies

$$
\begin{aligned}
\sup _{B}\left(\frac{1}{|B|} \int_{B} \nu_{\vec{w}}(x)^{q} d x\right)^{1 / q} \\
\quad \times \prod_{i=1}^{m}\left(\frac{1}{|B|} \int_{B} w_{i}(x)^{-p_{i}^{\prime}} d x\right)^{1 / p_{i}^{\prime}}<\infty .
\end{aligned}
$$

When $p_{i}=1,\left((1 /|B|) \int_{B} w_{i}(x)^{-p_{i}^{\prime}} d x\right)^{1 / p_{i}^{\prime}}$ is understood as inf $\left._{x \in B} w_{i}(x)\right)^{-1}$.

Given a ball $B$ and $\lambda>0, \lambda B$ denotes the ball with the same center as $B$ whose radius is $\lambda$ times that of $B$. For a given weight function $w$ and a measurable set $E$, we also denote the Lebesgue measure of $E$ by $|E|$ and the weighted measure of $E$ by $w(E)$, where $w(E)=\int_{E} w(x) d x$.

Lemma 5 (see [37]). Let $w \in A_{p}$ with $1 \leq p<\infty$. Then, for any ball $B$, there exists an absolute constant $C>0$ such that

$$
w(2 B) \leq C w(B)
$$

Lemma 6 (see [38]). Let $w \in A_{\infty}$. Then for all balls $B \subseteq \mathbb{R}^{n}$, the following reverse Jensen inequality holds:

$$
\int_{B} w(x) d x \leq C|B| \cdot \exp \left(\frac{1}{|B|} \int_{B} \log w(x) d x\right) .
$$

Lemma 7 (see [37]). Let $w \in A_{\infty}$. Then, for all balls $B$ and all measurable subsets $E$ of $B$, there exists $\delta>0$ such that

$$
\frac{w(E)}{w(B)} \leq C\left(\frac{|E|}{|B|}\right)^{\delta}
$$

Lemma 8 (see [9]). Let $p_{1}, \ldots, p_{m} \in[1, \infty)$, and let $1 / p=$ $\sum_{k=1}^{m} 1 / p_{k}$. Then $\vec{w}=\left(w_{1}, \ldots, w_{m}\right) \in A_{\vec{P}}$ if and only if

$$
\begin{gathered}
v_{\vec{w}} \in A_{m p}, \\
w_{i}^{1-p_{i}^{\prime}} \in A_{m p_{i}^{\prime}}, \quad i=1, \ldots, m,
\end{gathered}
$$

where $v_{\vec{w}}=\prod_{i=1}^{m} w_{i}^{p / p_{i}}$ and the condition $w_{i}^{1-p_{i}^{\prime}} \in A_{m p_{i}^{\prime}}$ in the case $p_{i}=1$ is understood as $w_{i}^{1 / m} \in A_{1}$. 
Lemma 9 (see $[17,18]$ ). Let $0<\alpha<m n$, and $p_{1}, \ldots, p_{m} \in$ $[1, \infty)$, let $1 / p=\sum_{k=1}^{m} 1 / p_{k}$, and let $1 / q=1 / p-\alpha / n$. If $\vec{w}=$ $\left(w_{1}, \ldots, w_{m}\right) \in A_{\vec{P}, q}$, then

$$
\begin{gathered}
\left(\nu_{\vec{w}}\right)^{q} \in A_{m q}, \\
w_{i}^{-p_{i}^{\prime}} \in A_{m p_{i}^{\prime}}, \quad i=1, \ldots, m
\end{gathered}
$$

where $v_{\vec{w}}=\prod_{i=1}^{m} w_{i}$.

Given a weight function $w$ on $\mathbb{R}^{n}$, for $0<p<\infty$, the weighted Lebesgue space $L^{p}(w)$ is defined as the set of all functions $f$ such that

$$
\|f\|_{L^{p}(w)}=\left(\int_{\mathbb{R}^{n}}|f(x)|^{p} w(x) d x\right)^{1 / p}<\infty .
$$

We also denote by $W L^{p}(w)$ the weighted weak space consisting of all measurable functions $f$ such that

$$
\|f\|_{W L^{p}(w)}=\sup _{\lambda>0} \lambda \cdot w\left(\left\{x \in \mathbb{R}^{n}:|f(x)|>\lambda\right\}\right)^{1 / p}<\infty .
$$

In 2009, Komori and Shirai [26] first defined the weighted Morrey spaces $L^{p, \kappa}(w)$ for $1 \leq p<\infty$. In order to deal with the multilinear case $m \geq 2$, we will define $L^{p, \kappa}(w)$ for all $0<$ $p<\infty$.

Definition 10. Let $0<p<\infty$, let $0<\kappa<1$, and let $w$ be a weight function on $\mathbb{R}^{n}$. Then the weighted Morrey space is defined by

$$
L^{p, \kappa}(w)=\left\{f \in L_{\mathrm{loc}}^{p}(w):\|f\|_{L^{p, \kappa}(w)}<\infty\right\},
$$

where

$$
\|f\|_{L^{p, \kappa}(w)}=\sup _{B}\left(\frac{1}{w(B)^{\kappa}} \int_{B}|f(x)|^{p} w(x) d x\right)^{1 / p}
$$

and the supremum is taken over all balls $B$ in $\mathbb{R}^{n}$.

Definition 11. Let $0<p<\infty$, let $0<\kappa<1$, and let $w$ be a weight function on $\mathbb{R}^{n}$. Then the weighted weak Morrey space is defined by

$$
W L^{p, \kappa}(w)=\left\{f \text { measurable }:\|f\|_{W L^{p, \kappa}(w)}<\infty\right\},
$$

where

$$
\|f\|_{W L^{p, \kappa}(w)}=\sup _{B} \sup _{\lambda>0} \frac{1}{w(B)^{\kappa / p}} \lambda \cdot w(\{x \in B:|f(x)|>\lambda\})^{1 / p} .
$$

Furthermore, in order to deal with the fractional order case, we need to consider the weighted Morrey spaces with two weights.

Definition 12. Let $0<p<\infty$ and $0<\kappa<1$. Then for two weights $u$ and $v$, the weighted Morrey space is defined by

$$
L^{p, \kappa}(u, v)=\left\{f \in L_{\mathrm{loc}}^{p}(u):\|f\|_{L^{p, \kappa}(u, v)}<\infty\right\},
$$

where

$$
\|f\|_{L^{p, \kappa}(u, v)}=\sup _{B}\left(\frac{1}{v(B)^{\kappa}} \int_{B}|f(x)|^{p} u(x) d x\right)^{1 / p} .
$$

Throughout this paper, we will use $C$ to denote a positive constant, which is independent of the main parameters and not necessarily the same at each occurrence. Moreover, we will denote the conjugate exponent of $p>1$ by $p^{\prime}=p /(p-1)$.

\section{Proofs of Theorems 1 and 2}

Before proving the main theorems of this section, we need to establish the following lemma.

Lemma 13. Let $m \geq 2$, let $p_{1}, \ldots, p_{m} \in[1, \infty)$, and let $p \in$ $(0, \infty)$ with $1 / p=\sum_{k=1}^{m} 1 / p_{k}$. Assume that $w_{1}, \ldots, w_{m} \in A_{\infty}$ and $\nu_{\vec{w}}=\prod_{i=1}^{m} w_{i}^{p / p_{i}} ;$ then, for any ball $B$, there exists a constant $C>0$ such that

$$
\prod_{i=1}^{m}\left(\int_{B} w_{i}(x) d x\right)^{p / p_{i}} \leq C \int_{B} \nu_{\vec{w}}(x) d x
$$

Proof. Since $w_{1}, \ldots, w_{m} \in A_{\infty}$, then, by using Lemma 6, we have

$$
\begin{aligned}
& \prod_{i=1}^{m}\left(\int_{B} w_{i}(x) d x\right)^{p / p_{i}} \\
& \quad \leq C \prod_{i=1}^{m}\left(|B| \cdot \exp \left(\frac{1}{|B|} \int_{B} \log w_{i}(x) d x\right)\right)^{p / p_{i}} \\
& \quad=C \prod_{i=1}^{m}\left(|B|^{p / p_{i}} \cdot \exp \left(\frac{1}{|B|} \int_{B} \log w_{i}(x)^{p / p_{i}} d x\right)\right) \\
& \quad=C \cdot(|B|)^{\sum_{i=1}^{m} p / p_{i}} \cdot \exp \left(\sum_{i=1}^{m} \frac{1}{|B|} \int_{B} \log w_{i}(x)^{p / p_{i}} d x\right) .
\end{aligned}
$$

Note that $\sum_{i=1}^{m} p / p_{i}=1$ and $\nu_{\vec{w}}(x)=\prod_{i=1}^{m} w_{i}(x)^{p / p_{i}}$. Then, by Jensen inequality, we obtain

$$
\begin{aligned}
& \prod_{i=1}^{m}\left(\int_{B} w_{i}(x) d x\right)^{p / p_{i}} \\
& \quad \leq C \cdot|B| \cdot \exp \left(\frac{1}{|B|} \int_{B} \log \nu_{\vec{w}}(x) d x\right) \\
& \quad \leq C \int_{B} \nu_{\vec{w}}(x) d x .
\end{aligned}
$$

We are done. 
Proof of Theorem 1. For any ball $B=B\left(x_{0}, r_{B}\right) \subseteq \mathbb{R}^{n}$ and letting $f_{i}=f_{i}^{0}+f_{i}^{\infty}$, where $f_{i}^{0}=f_{i} \chi_{2 B}, i=1, \ldots, m$, and $\chi_{2 B}$ denotes the characteristic function of $2 B$, then we write

$$
\begin{aligned}
\prod_{i=1}^{m} f_{i}\left(y_{i}\right) & =\prod_{i=1}^{m}\left(f_{i}^{0}\left(y_{i}\right)+f_{i}^{\infty}\left(y_{i}\right)\right) \\
& =\sum_{\alpha_{1}, \ldots, \alpha_{m} \in\{0, \infty\}} f_{1}^{\alpha_{1}}\left(y_{1}\right) \cdots f_{m}^{\alpha_{m}}\left(y_{m}\right) \\
& =\prod_{i=1}^{m} f_{i}^{0}\left(y_{i}\right)+\sum^{\prime} f_{1}^{\alpha_{1}}\left(y_{1}\right) \cdots f_{m}^{\alpha_{m}}\left(y_{m}\right),
\end{aligned}
$$

where each term of $\sum^{\prime}$ contains at least one $\alpha_{i} \neq 0$. Since $T$ is an $m$-linear operator, then we have

$$
\begin{aligned}
& \frac{1}{v_{\vec{w}}(B)^{\kappa / p}}\left(\int_{B}\left|T\left(f_{1}, \ldots, f_{m}\right)(x)\right|^{p} v_{\vec{w}}(x) d x\right)^{1 / p} \\
& \leq \frac{1}{v_{\vec{w}}(B)^{\kappa / p}}\left(\int_{B}\left|T\left(f_{1}^{0}, \ldots, f_{m}^{0}\right)(x)\right|^{p} v_{\vec{w}}(x) d x\right)^{1 / p} \\
& \quad+\sum^{\prime} \frac{1}{v_{\vec{w}}(B)^{\kappa / p}}\left(\int_{B}\left|T\left(f_{1}^{\alpha_{1}}, \ldots, f_{m}^{\alpha_{m}}\right)(x)\right|^{p} v_{\vec{w}}(x) d x\right)^{1 / p} \\
& =I^{0}+\sum^{\prime} I^{\alpha_{1}, \ldots, \alpha_{m}} .
\end{aligned}
$$

In view of Lemma 8, we have that $\nu_{\vec{w}} \in A_{m p}$. Applying Theorem A and Lemmas 13 and 5, we get

$$
\begin{aligned}
I^{0} & \leq C \cdot \frac{1}{v_{\vec{w}}(B)^{\kappa / p}} \prod_{i=1}^{m}\left(\int_{2 B}\left|f_{i}(x)\right|^{p_{i}} w_{i}(x) d x\right)^{1 / p_{i}} \\
& \leq C \prod_{i=1}^{m}\left\|f_{i}\right\|_{L^{p_{i}, \kappa}\left(w_{i}\right)} \cdot \frac{\prod_{i=1}^{m} w_{i}(2 B)^{\kappa / p_{i}}}{v_{\vec{w}}(B)^{\kappa / p}} \\
& \leq C \prod_{i=1}^{m}\left\|f_{i}\right\|_{L^{p_{i}, \kappa}\left(w_{i}\right)} \cdot \frac{v_{\vec{w}}(2 B)^{\kappa / p}}{v_{\vec{w}}(B)^{\kappa / p}} \\
& \leq C \prod_{i=1}^{m}\left\|f_{i}\right\|_{L^{p_{i}, \kappa}\left(w_{i}\right)} \cdot
\end{aligned}
$$

For the other terms, let us first consider the case when $\alpha_{1}=$ $\cdots=\alpha_{m}=\infty$. By the size condition, for any $x \in B$, we obtain

$$
\begin{aligned}
& \left|T\left(f_{1}^{\infty}, \ldots, f_{m}^{\infty}\right)(x)\right| \\
& \quad \leq C \int_{\left(\mathbb{R}^{n}\right)^{m} \backslash(2 B)^{m}} \frac{\left|f_{1}\left(y_{1}\right) \cdots f_{m}\left(y_{m}\right)\right|}{\left(\left|x-y_{1}\right|+\cdots+\left|x-y_{m}\right|\right)^{m n}} d y_{1} \cdots d y_{m} \\
& \quad \leq C \sum_{j=1}^{\infty} \int_{\left(2^{j+1} B\right)^{m} \backslash\left(2^{j} B\right)^{m}} \frac{\left|f_{1}\left(y_{1}\right) \cdots f_{m}\left(y_{m}\right)\right|}{\left(\left|x-y_{1}\right|+\cdots+\mid x-y_{m}\right)^{m n}} d y_{1} \cdots d y_{m} \\
& \quad \leq C \sum_{j=1}^{\infty} \prod_{i=1}^{m} \int_{2^{j+1} B \backslash 2^{j} B} \frac{\left|f_{i}\left(y_{i}\right)\right|}{\left|x-y_{i}\right|^{n}} d y_{i} \\
& \quad \leq C \sum_{j=1}^{\infty} \prod_{i=1}^{m} \frac{1}{\left|2^{j+1} B\right|} \int_{2^{j+1} B}\left|f_{i}\left(y_{i}\right)\right| d y_{i},
\end{aligned}
$$

where we have used the notation $E^{m}=E \times \cdots \times E$. Furthermore, by using Hölder's inequality, the multiple $A_{\vec{P}}$ condition, and Lemma 13, we deduce that

$$
\begin{aligned}
& \left|T\left(f_{1}^{\infty}, \ldots, f_{m}^{\infty}\right)(x)\right| \\
& \leq C \sum_{j=1}^{\infty} \prod_{i=1}^{m} \frac{1}{\left|2^{j+1} B\right|}\left(\int_{2^{j+1} B}\left|f_{i}\left(y_{i}\right)\right|^{p_{i}} w_{i}\left(y_{i}\right) d y_{i}\right)^{1 / p_{i}} \\
& \quad \times\left(\int_{2^{j+1} B} w_{i}\left(y_{i}\right)^{1-p_{i}^{\prime}} d y_{i}\right)^{1 / p_{i}^{\prime}} \\
& \leq C \sum_{j=1}^{\infty} \frac{1}{\left.2^{j+1} B\right|^{m}} \cdot \frac{\left|2^{j+1} B\right|^{1 / p+\sum_{i=1}^{m}\left(1-1 / p_{i}\right)}}{v_{\vec{w}}\left(2^{j+1} B\right)^{1 / p}} \\
& \quad \times \prod_{i=1}^{m}\left(\left\|f_{i}\right\|_{L^{p_{i}, \kappa}\left(w_{i}\right)} w_{i}\left(2^{j+1} B\right)^{\kappa / p_{i}}\right) \\
& \leq C \prod_{i=1}^{m}\left\|f_{i}\right\|_{L^{p_{i}, \kappa}\left(w_{i}\right)} \cdot \sum_{j=1}^{\infty}\left(\frac{\prod_{i=1}^{m} w_{i}\left(2^{j+1} B\right)^{\kappa / p_{i}}}{v_{\vec{w}}\left(2^{j+1} B\right)^{1 / p}}\right) \\
& \leq C \prod_{i=1}^{m}\left\|f_{i}\right\|_{L^{p_{i}, \kappa}\left(w_{i}\right)} \cdot \sum_{j=1}^{\infty} v_{\vec{w}}\left(2^{j+1} B\right)^{(\kappa-1) / p} .
\end{aligned}
$$

Since $v_{\vec{w}} \in A_{m p} \subset A_{\infty}$, then it follows directly from Lemma 7 that

$$
\frac{v_{\vec{w}}(B)}{v_{\vec{w}}\left(2^{j+1} B\right)} \leq C\left(\frac{|B|}{\left|2^{j+1} B\right|}\right)^{\delta} .
$$

Hence,

$$
\begin{aligned}
I^{\infty, \ldots, \infty} & \leq v_{\vec{w}}(B)^{(1-\kappa) / p}\left|T\left(f_{1}^{\infty}, \ldots, f_{m}^{\infty}\right)(x)\right| \\
& \leq C \prod_{i=1}^{m}\left\|f_{i}\right\|_{L^{p_{i}, \kappa}\left(w_{i}\right)} \cdot \sum_{j=1}^{\infty} \frac{v_{\vec{w}}(B)^{(1-\kappa) / p}}{v_{\vec{w}}\left(2^{j+1} B\right)^{(1-\kappa) / p}} \\
& \leq C \prod_{i=1}^{m}\left\|f_{i}\right\|_{L^{p_{i}, \kappa}\left(w_{i}\right)} \cdot \sum_{j=1}^{\infty}\left(\frac{|B|}{\left|2^{j+1} B\right|}\right)^{\delta(1-\kappa) / p} \\
& \leq C \prod_{i=1}^{m}\left\|f_{i}\right\|_{L^{p_{i}, \kappa}\left(w_{i}\right)},
\end{aligned}
$$

where the last inequality holds since $0<\kappa<1$ and $\delta>0$. We now consider the case where exactly $\ell$ of the $\alpha_{i}$ are $\infty$ for some $1 \leq \ell<m$. We only give the arguments for one of these cases. The rest are similar and can easily be obtained from the arguments below by permuting the indices. Using the size condition again, we deduce that, for any $x \in B$,

$$
\begin{aligned}
& \left|T\left(f_{1}^{\infty}, \ldots, f_{\ell}^{\infty}, f_{\ell+1}^{0}, \ldots, f_{m}^{0}\right)(x)\right| \\
& \leq C \int_{\left(\mathbb{R}^{n}\right)^{\ell} \backslash(2 B)^{\ell}} \int_{(2 B)^{m-\ell}} \frac{\left|f_{1}\left(y_{1}\right) \cdots f_{m}\left(y_{m}\right)\right|}{\left(\left|x-y_{1}\right|+\cdots+\left|x-y_{m}\right|\right)^{m n}} \\
& \quad \times d y_{1} \cdots d y_{m}
\end{aligned}
$$




$$
\begin{aligned}
\leq & C \prod_{i=\ell+1}^{m} \int_{2 B}\left|f_{i}\left(y_{i}\right)\right| d y_{i} \\
& \times \sum_{j=1}^{\infty} \frac{1}{\left|2^{j+1} B\right|^{m}} \\
& \times \int_{\left(2^{j+1} B\right)^{\ell} \backslash\left(2^{j} B\right)^{\ell}}\left|f_{1}\left(y_{1}\right) \cdots f_{\ell}\left(y_{\ell}\right)\right| d y_{1} \cdots d y_{\ell} \\
\leq & C \prod_{i=\ell+1}^{m} \int_{2 B}\left|f_{i}\left(y_{i}\right)\right| d y_{i} \\
& \times \sum_{j=1}^{\infty} \frac{1}{\left|2^{j+1} B\right|^{m}} \prod_{i=1}^{\ell} \int_{2^{j+1} B \backslash 2^{j} B}\left|f_{i}\left(y_{i}\right)\right| d y_{i} \\
\leq & C \sum_{j=1}^{\infty} \prod_{i=1}^{m} \frac{1}{\left|2^{j+1} B\right|} \int_{2^{j+1} B}\left|f_{i}\left(y_{i}\right)\right| d y_{i},
\end{aligned}
$$

and we arrive at the expression considered in the previous case. So for any $x \in B$, we also have

$$
\begin{aligned}
& \left|T\left(f_{1}^{\infty}, \ldots, f_{\ell}^{\infty}, f_{\ell+1}^{0}, \ldots, f_{m}^{0}\right)(x)\right| \\
& \quad \leq C \prod_{i=1}^{m}\left\|f_{i}\right\|_{L^{p_{i}, \kappa}\left(w_{i}\right)} \cdot \sum_{j=1}^{\infty} v_{\vec{w}}\left(2^{j+1} B\right)^{(\kappa-1) / p} .
\end{aligned}
$$

Therefore, by the inequality (42) and the above pointwise inequality, we have

$$
\begin{aligned}
& I^{\alpha_{1}, \ldots, \alpha_{m}} \\
& \quad \leq v_{\vec{w}}(B)^{(1-\kappa) / p}\left|T\left(f_{1}^{\infty}, \ldots, f_{\ell}^{\infty}, f_{\ell+1}^{0}, \ldots, f_{m}^{0}\right)(x)\right| \\
& \quad \leq C \prod_{i=1}^{m}\left\|f_{i}\right\|_{L^{p^{i}, \kappa}\left(w_{i}\right)} \cdot \sum_{j=1}^{\infty} \frac{v_{\vec{w}}(B)^{(1-\kappa) / p}}{v_{\vec{w}}\left(2^{j+1} B\right)^{(1-\kappa) / p}} \\
& \quad \leq C \prod_{i=1}^{m}\left\|f_{i}\right\|_{L^{p_{i}, \kappa}\left(w_{i}\right)} \cdot \sum_{j=1}^{\infty}\left(\frac{|B|}{\left|2^{j+1} B\right|}\right)^{\delta(1-\kappa) / p} \\
& \quad \leq C \prod_{i=1}^{m}\left\|f_{i}\right\|_{L^{p_{i}, \kappa}\left(w_{i}\right)} .
\end{aligned}
$$

Combining the above estimates and then taking the supremum over all balls $B \subseteq \mathbb{R}^{n}$, we complete the proof of Theorem 1.

Proof of Theorem 2. For any ball $B=B\left(x_{0}, r_{B}\right) \subseteq \mathbb{R}^{n}$ and decomposing $f_{i}=f_{i}^{0}+f_{i}^{\infty}$, where $f_{i}^{0}=f_{i} \chi_{2 B}, i=1, \ldots, m$, then, for any given $\lambda>0$, we can write

$$
\begin{aligned}
\nu_{\vec{w}}(\{x \in & \left.\left.B:\left|T\left(f_{1}, \ldots, f_{m}\right)\right|>\lambda\right\}\right)^{1 / p} \\
\leq & \nu_{\vec{w}}\left(\left\{x \in B:\left|T\left(f_{1}^{0}, \ldots, f_{m}^{0}\right)\right|>\frac{\lambda}{2^{m}}\right\}\right)^{1 / p} \\
& +\sum^{\prime} v_{\vec{w}}\left(\left\{x \in B:\left|T\left(f_{1}^{\alpha_{1}}, \ldots, f_{m}^{\alpha_{m}}\right)\right|>\frac{\lambda}{2^{m}}\right\}\right)^{1 / p} \\
= & I_{*}^{0}+\sum^{\prime} I_{*}^{\alpha_{1}, \ldots, \alpha_{m}},
\end{aligned}
$$

where each term of $\sum^{\prime}$ contains at least one $\alpha_{i} \neq 0$. By Lemma 8 again, we know that $\nu_{\vec{w}} \in A_{m p}$ with $1 \leq m p<\infty$. Applying Theorem $\mathrm{B}$ and Lemmas 13 and 5, we have

$$
\begin{aligned}
I_{*}^{0} & \leq \frac{C}{\lambda} \prod_{i=1}^{m}\left(\int_{2 B}\left|f_{i}(x)\right|^{p_{i}} w_{i}(x) d x\right)^{1 / p_{i}} \\
& \leq \frac{C \cdot \prod_{i=1}^{m} w_{i}(2 B)^{\kappa / p_{i}}}{\lambda} \prod_{i=1}^{m}\left\|f_{i}\right\|_{L^{p_{i}, \kappa}\left(w_{i}\right)} \\
& \leq \frac{C \cdot v_{\vec{w}}(2 B)^{\kappa / p}}{\lambda} \prod_{i=1}^{m}\left\|f_{i}\right\|_{L^{p_{i}, \kappa}\left(w_{i}\right)} \\
& \leq \frac{C \cdot v_{\vec{w}}(B)^{\kappa / p}}{\lambda} \prod_{i=1}^{m}\left\|f_{i}\right\|_{L^{p_{i}, \kappa}\left(w_{i}\right)} .
\end{aligned}
$$

In the proof of Theorem 1, we have already showed the following pointwise estimate (see (40) and (44)). Consider

$$
\left|T\left(f_{1}^{\alpha_{1}}, \ldots, f_{m}^{\alpha_{m}}\right)(x)\right| \leq C \sum_{j=1}^{\infty} \prod_{i=1}^{m} \frac{1}{\left|2^{j+1} B\right|} \int_{2^{j+1} B}\left|f_{i}\left(y_{i}\right)\right| d y_{i}
$$

Without loss of generality, we may assume that $p_{1}=\cdots=$ $p_{\ell}=\min \left\{p_{1}, \ldots, p_{m}\right\}=1$ and $p_{\ell+1}, \ldots, p_{m}>1$. Using Hölder's inequality, the multiple $A_{\vec{P}}$ condition, and Lemma 13, we obtain

$$
\begin{aligned}
&\left|T\left(f_{1}^{\alpha_{1}}, \ldots, f_{m}^{\alpha_{m}}\right)(x)\right| \\
& \leq C \sum_{j=1}^{\infty} \prod_{i=1}^{\ell} \frac{1}{\left|2^{j+1} B\right|} \int_{2^{j+1} B}\left|f_{i}\left(y_{i}\right)\right| d y_{i} \\
& \times \prod_{i=\ell+1}^{m} \frac{1}{\left|2^{j+1} B\right|} \int_{2^{j+1} B}\left|f_{i}\left(y_{i}\right)\right| d y_{i} \\
& \leq C \sum_{j=1}^{\infty} \prod_{i=1}^{\ell} \frac{1}{\left|2^{j+1} B\right|} \int_{2^{j+1} B}\left|f_{i}\left(y_{i}\right)\right| w_{i}\left(y_{i}\right) d y_{i} \\
& \times \prod_{i=\ell+1}^{m} \frac{1}{\left|2^{j+1} B\right|}\left(\int_{2^{j+1} B}\left|f_{i}\left(y_{i}\right)\right|^{p_{i}} w_{i}\left(y_{i}\right) d y_{i}\right)^{1 / p_{i}} \\
& \leq\left.C \prod_{i=1}^{m} \| w_{i}\left(y_{i}\right)\right)^{-1} \\
& \times\left(\int_{L^{p_{i}, \kappa}\left(w_{i}\right)} \sum_{j=1}^{\infty} v_{\vec{w}}\left(2^{j+1} B\right)^{(\kappa-1) / p} .\right.
\end{aligned}
$$

Observe that $\nu_{\vec{w}} \in A_{m p}$ with $1 \leq m p<\infty$. Thus, it follows from the inequality (42) that, for any $x \in B$,

$$
\begin{aligned}
& \left|T\left(f_{1}^{\alpha_{1}}, \ldots, f_{m}^{\alpha_{m}}\right)(x)\right| \\
& \quad=C \prod_{i=1}^{m}\left\|f_{i}\right\|_{L^{p_{i}, \kappa}\left(w_{i}\right)} \cdot \frac{1}{v_{\vec{w}}(B)^{(1-\kappa) / p}} \sum_{j=1}^{\infty} \frac{v_{\vec{w}}(B)^{(1-\kappa) / p}}{\nu_{\vec{w}}\left(2^{j+1} B\right)^{(1-\kappa) / p}}
\end{aligned}
$$




$$
\begin{aligned}
& \leq C \prod_{i=1}^{m}\left\|f_{i}\right\|_{L^{p_{i}, \kappa}\left(w_{i}\right)} \cdot \frac{1}{v_{\vec{w}}(B)^{(1-\kappa) / p}} \sum_{j=1}^{\infty}\left(\frac{|B|}{\left|2^{j+1} B\right|}\right)^{\delta(1-\kappa) / p} \\
& \leq C \prod_{i=1}^{m}\left\|f_{i}\right\|_{L^{p_{i}, \kappa}\left(w_{i}\right)} \cdot \frac{1}{v_{\vec{w}}(B)^{(1-\kappa) / p}} .
\end{aligned}
$$

If $\left\{x \in B:\left|T\left(f_{1}^{\alpha_{1}}, \ldots, f_{m}^{\alpha_{m}}\right)(x)\right|>\lambda / 2^{m}\right\}=\varnothing$, then the inequality

$$
I_{*}^{\alpha_{1}, \ldots, \alpha_{m}} \leq \frac{C \cdot \nu_{\vec{w}}(B)^{\kappa / p}}{\lambda} \prod_{i=1}^{m}\left\|f_{i}\right\|_{L^{p_{i}, \kappa}\left(w_{i}\right)}
$$

holds trivially. Now, if instead we suppose that $\{x \in B$ : $\left.\left|T\left(f_{1}^{\alpha_{1}}, \ldots, f_{m}^{\alpha_{m}}\right)(x)\right|>\lambda / 2^{m}\right\} \neq \varnothing$, then, by the pointwise inequality (51), we have

$$
\lambda<C \prod_{i=1}^{m}\left\|f_{i}\right\|_{L^{p_{i}, \kappa}\left(w_{i}\right)} \cdot \frac{1}{v_{\vec{w}}(B)^{(1-\kappa) / p}}
$$

which is equivalent to

$$
\nu_{\vec{w}}(B)^{1 / p} \leq \frac{C \cdot \nu_{\vec{w}}(B)^{\kappa / p}}{\lambda} \prod_{i=1}^{m}\left\|f_{i}\right\|_{L^{p_{i}, \kappa}\left(w_{i}\right)}
$$

Therefore,

$$
I_{*}^{\alpha_{1}, \ldots, \alpha_{m}} \leq \nu_{\vec{w}}(B)^{1 / p} \leq \frac{C \cdot \nu_{\vec{w}}(B)^{\kappa / p}}{\lambda} \prod_{i=1}^{m}\left\|f_{i}\right\|_{L^{p_{i}, \kappa}\left(w_{i}\right)}
$$

Summing up all the above estimates and then taking the supremum over all balls $B \subseteq \mathbb{R}^{n}$ and all $\lambda>0$, we complete the proof of Theorem 2 .

By using Hölder's inequality, it is easy to check that if each $w_{i}$ is in $A_{p_{i}}$, then

$$
\prod_{i=1}^{m} A_{p_{i}} \subset A_{\vec{P}}
$$

and this inclusion is strict (see [9]). Thus, as direct consequences of Theorems 1 and 2, we immediately obtain the following.

Corollary 14. Let $m \geq 2$, and let $T$ be an $m$-linear CalderónZygmund operator. If $p_{1}, \ldots, p_{m} \in(1, \infty)$ and $p \in(0, \infty)$ with $1 / p=\sum_{k=1}^{m} 1 / p_{k}$ and $\vec{w}=\left(w_{1}, \ldots, w_{m}\right) \in \prod_{i=1}^{m} A_{p_{i}}$, then, for any $0<\kappa<1$, there exists a constant $C>0$ independent of $\vec{f}=\left(f_{1}, \ldots, f_{m}\right)$ such that

$$
\|T(\vec{f})\|_{L^{p, \kappa}\left(v_{\vec{w}}\right)} \leq C \prod_{i=1}^{m}\left\|f_{i}\right\|_{L^{p_{i}, \kappa}\left(w_{i}\right)},
$$

where $\nu_{\vec{w}}=\prod_{i=1}^{m} w_{i}^{p / p_{i}}$.
Corollary 15. Let $m \geq 2$ and let $T$ be an $m$-linear CalderónZygmund operator. If $p_{1}, \ldots, p_{m} \in[1, \infty), \min \left\{p_{1}, \ldots, p_{m}\right\}=$ 1 and $p \in(0, \infty)$ with $1 / p=\sum_{k=1}^{m} 1 / p_{k}$, and $\vec{w}=$ $\left(w_{1}, \ldots, w_{m}\right) \in \prod_{i=1}^{m} A_{p_{i}}$, then, for any $0<\kappa<1$, there exists a constant $C>0$ independent of $\vec{f}=\left(f_{1}, \ldots, f_{m}\right)$ such that

$$
\|T(\vec{f})\|_{W L^{p, \kappa}\left(v_{\vec{w}}\right)} \leq C \prod_{i=1}^{m}\left\|f_{i}\right\|_{L^{p_{i}, \kappa}\left(w_{i}\right)},
$$

where $\nu_{\vec{w}}=\prod_{i=1}^{m} w_{i}^{p / p_{i}}$.

\section{Proofs of Theorems 3 and 4}

Following along the same lines as those of Lemma 13, we can also show the following result, which plays an important role in our proofs of Theorems 3 and 4.

Lemma 16. Let $m \geq 2, q_{1}, \ldots, q_{m} \in[1, \infty)$ and $q \in(0, \infty)$ with $1 / q=\sum_{k=1}^{m} 1 / q_{k}$. Assume that $w_{1}^{q_{1}}, \ldots, w_{m}^{q_{m}} \in A_{\infty}$ and $\nu_{\vec{w}}=\prod_{i=1}^{m} w_{i}$; then, for any ball $B$, there exists a constant $C>0$ such that

$$
\prod_{i=1}^{m}\left(\int_{B} w_{i}^{q_{i}}(x) d x\right)^{q / q_{i}} \leq C \int_{B} \nu_{\vec{w}}(x)^{q} d x .
$$

Proof of Theorem 3. Arguing as in the proof of Theorem 1, fix a ball $B=B\left(x_{0}, r_{B}\right) \subseteq \mathbb{R}^{n}$ and decompose $f_{i}=f_{i}^{0}+f_{i}^{\infty}$, where $f_{i}^{0}=f_{i} \chi_{2 B}, i=1, \ldots, m$. Since $I_{\alpha}$ is an $m$-linear operator, then we have

$$
\begin{aligned}
& \frac{1}{\nu_{\vec{w}}^{q}(B)^{\kappa / p}}\left(\int_{B}\left|I_{\alpha}\left(f_{1}, \ldots, f_{m}\right)(x)\right|^{q} \nu_{\vec{w}}(x)^{q} d x\right)^{1 / q} \\
& \leq \frac{1}{\nu_{\vec{w}}^{q}(B)^{\kappa / p}}\left(\int_{B}\left|I_{\alpha}\left(f_{1}^{0}, \ldots, f_{m}^{0}\right)(x)\right|^{q} \nu_{\vec{w}}(x)^{q} d x\right)^{1 / q} \\
& \quad+\sum^{\prime} \frac{1}{v_{\vec{w}}^{q}(B)^{\kappa / p}}\left(\int_{B}\left|I_{\alpha}\left(f_{1}^{\alpha_{1}}, \ldots, f_{m}^{\alpha_{m}}\right)(x)\right|^{q} \nu_{\vec{w}}(x)^{q} d x\right)^{1 / q} \\
& =J^{0}+\sum^{\prime} J^{\alpha_{1}, \ldots, \alpha_{m}},
\end{aligned}
$$

where each term of $\sum^{\prime}$ contains at least one $\alpha_{i} \neq 0$. In view of Lemma 9 , we can see that $\left(\nu_{\vec{w}}\right)^{q} \in A_{m q}$. Using Theorem $\mathrm{C}$ and Lemmas 16 and 5, we get

$$
\begin{aligned}
J^{0} & \leq C \cdot \frac{1}{v_{\vec{w}}^{q}(B)^{\kappa / p}} \prod_{i=1}^{m}\left(\int_{2 B}\left|f_{i}(x)\right|^{p_{i}} w_{i}(x)^{p_{i}} d x\right)^{1 / p_{i}} \\
& \leq C \prod_{i=1}^{m}\left\|f_{i}\right\|_{L^{p_{i}, \kappa p_{i} q / p q_{i}}\left(w_{i}^{p_{i}}, w_{i}^{q_{i}}\right)} \cdot \frac{\prod_{i=1}^{m} w_{i}^{q_{i}}(2 B)^{\kappa q / p q_{i}}}{\nu_{\vec{w}}^{q}(B)^{\kappa / p}} \\
& =C \prod_{i=1}^{m}\left\|f_{i}\right\|_{L^{p_{i}, \kappa p_{i} q / p q_{i}}\left(w_{i}^{p_{i}}, w_{i}^{q_{i}}\right)} \cdot \frac{\left(\prod_{i=1}^{m} w_{i}^{q_{i}}(2 B)^{q / q_{i}}\right)^{\kappa / p}}{v_{\vec{w}}^{q}(B)^{\kappa / p}} \\
& \leq C \prod_{i=1}^{m}\left\|f_{i}\right\|_{L^{p_{i}, \kappa p_{i} q / p q_{i}}\left(w_{i}^{p_{i}}, w_{i}^{q_{i}}\right)} \cdot \frac{\nu_{\vec{w}}^{q}(2 B)^{\kappa / p}}{\nu_{\vec{w}}^{q}(B)^{\kappa / p}} \\
& \leq C \prod_{i=1}^{m}\left\|f_{i}\right\|_{L^{p_{i}, \kappa p_{i} q / p q_{i}}\left(w_{i}^{p_{i}}, w_{i}^{q_{i}}\right)}
\end{aligned}
$$


For the other terms, let us first deal with the case when $\alpha_{1}=$ $\cdots=\alpha_{m}=\infty$. By the definition of $I_{\alpha}$, for any $x \in B$, we obtain

$$
\begin{aligned}
& \left|I_{\alpha}\left(f_{1}^{\infty}, \ldots, f_{m}^{\infty}\right)(x)\right| \\
& \quad=\int_{\left(\mathbb{R}^{n}\right)^{m} \backslash(2 B)^{m}} \frac{\left|f_{1}\left(y_{1}\right) \cdots f_{m}\left(y_{m}\right)\right|}{\left(\left|x-y_{1}\right|+\cdots+\left|x-y_{m}\right|\right)^{m n-\alpha}} d y_{1} \cdots d y_{m} \\
& \quad=\sum_{j=1}^{\infty} \int_{\left(2^{j+1} B\right)^{m} \backslash\left(2^{j} B\right)^{m}} \frac{\left|f_{1}\left(y_{1}\right) \cdots f_{m}\left(y_{m}\right)\right|}{\left(\left|x-y_{1}\right|+\cdots+\left|x-y_{m}\right|\right)^{m n-\alpha}} \\
& \quad \leq C \sum_{j=1}^{\infty} \prod_{i=1}^{m} \int_{2^{j+1} B \backslash 2^{j} B} \frac{\left|f_{i}\left(y_{i}\right)\right|}{\left|x-y_{i}\right|^{n-\alpha / m}} d y_{i} \\
& \quad \leq C \sum_{j=1}^{\infty} \prod_{i=1}^{m} \frac{1}{\left|2^{j+1} B\right|^{1-\alpha / m n}} \int_{2^{j+1} B}\left|f_{i}\left(y_{i}\right)\right| d y_{i} .
\end{aligned}
$$

Moreover, by using Hölder's inequality, the multiple $A_{\vec{P}, q}$ condition, and Lemma 16, we deduce that

$$
\begin{aligned}
& \left|I_{\alpha}\left(f_{1}^{\infty}, \ldots, f_{m}^{\infty}\right)(x)\right| \\
& \leq C \sum_{j=1}^{\infty} \prod_{i=1}^{m} \frac{1}{\left|2^{j+1} B\right|^{1-\alpha / m n}} \\
& \times\left(\int_{2^{j+1} B}\left|f_{i}\left(y_{i}\right)\right|^{p_{i}} w_{i}\left(y_{i}\right)^{p_{i}} d y_{i}\right)^{1 / p_{i}} \\
& \times\left(\int_{2^{j+1} B} w_{i}\left(y_{i}\right)^{-p_{i}^{\prime}} d y_{i}\right)^{1 / p_{i}^{\prime}} \\
& \leq C \sum_{j=1}^{\infty} \frac{1}{\left|2^{j+1} B\right|^{m-\alpha / n}} \cdot \frac{\left|2^{j+1} B\right|^{1 / q+\sum_{i=1}^{m}\left(1-1 / p_{i}\right)}}{\nu_{\vec{w}}^{q}\left(2^{j+1} B\right)^{1 / q}} \\
& \times \prod_{i=1}^{m}\left(\left\|f_{i}\right\|_{L^{p_{i}, \kappa p_{i} q / p q_{i}}\left(w_{i}^{p_{i}}, w_{i}^{q_{i}}\right)} w_{i}^{q_{i}}\left(2^{j+1} B\right)^{\kappa q / p q_{i}}\right) \\
& \leq C \prod_{i=1}^{m}\left\|f_{i}\right\|_{L^{p_{i}, \kappa p_{i} q / p q_{i}}\left(w_{i}^{p_{i}}, w_{i}^{q_{i}}\right)} \\
& \cdot \sum_{j=1}^{\infty}\left[\frac{\left(\prod_{i=1}^{m} w_{i}^{q_{i}}\left(2^{j+1} B\right)^{q / q_{i}}\right)^{\kappa / p}}{v_{\vec{w}}^{q}\left(2^{j+1} B\right)^{1 / q}}\right] \\
& \leq C \prod_{i=1}^{m}\left\|f_{i}\right\|_{L^{p_{i}, \kappa p_{i} q / p q_{i}}\left(w_{i}^{p_{i}}, w_{i}^{q_{i}}\right)} \cdot \sum_{j=1}^{\infty} \nu_{\vec{w}}^{q}\left(2^{j+1} B\right)^{\kappa / p-1 / q} .
\end{aligned}
$$

Since $\left(\nu_{\vec{w}}\right)^{q} \in A_{m q} \subset A_{\infty}$, then it follows immediately from Lemma 7 that

$$
\frac{v_{\vec{w}}^{q}(B)}{v_{\vec{w}}^{q}\left(2^{j+1} B\right)} \leq C\left(\frac{|B|}{\left|2^{j+1} B\right|}\right)^{\delta^{\prime}}
$$

Hence,

$$
\begin{aligned}
J^{\infty, \ldots, \infty} & \leq \nu_{\vec{w}}^{q}(B)^{1 / q-\kappa / p}\left|I_{\alpha}\left(f_{1}^{\infty}, \ldots, f_{m}^{\infty}\right)(x)\right| \\
& \leq C \prod_{i=1}^{m}\left\|f_{i}\right\|_{L^{p_{i}, \kappa p_{i} q / p q_{i}}\left(w_{i}^{p_{i}}, w_{i}^{q_{i}}\right)} \cdot \sum_{j=1}^{\infty} \frac{\nu_{\vec{w}}^{q}(B)^{1 / q-\kappa / p}}{\nu_{\vec{w}}^{q}\left(2^{j+1} B\right)^{1 / q-\kappa / p}} \\
& \leq C \prod_{i=1}^{m}\left\|f_{i}\right\|_{L^{p_{i}, \kappa p_{i} q / p q_{i}}\left(w_{i}^{p_{i}}, w_{i}^{q_{i}}\right)} \cdot \sum_{j=1}^{\infty}\left(\frac{|B|}{\left|2^{j+1} B\right|}\right)^{\delta^{\prime}(1 / q-\kappa / p)} \\
& \leq C \prod_{i=1}^{m}\left\|f_{i}\right\|_{L^{p_{i}, \kappa p_{i} q / p q_{i}}\left(w_{i}^{p_{i}}, w_{i}^{q_{i}}\right)},
\end{aligned}
$$

where in the last inequality we have used the fact that $0<\kappa<$ $p / q$ and $\delta^{\prime}>0$. We now consider the case where exactly $\ell$ of the $\alpha_{i}$ are $\infty$ for some $1 \leq \ell<m$. We only give the arguments for one of these cases. The rest are similar and can easily be obtained from the arguments below by permuting the indices. Using the definition of $I_{\alpha}$ again, we can see that, for any $x \in B$,

$$
\begin{aligned}
& \left|I_{\alpha}\left(f_{1}^{\infty}, \ldots, f_{\ell}^{\infty}, f_{\ell+1}^{0}, \ldots, f_{m}^{0}\right)(x)\right| \\
& =\int_{\left(\mathbb{R}^{n}\right)^{\ell} \backslash(2 B)^{\ell}} \int_{(2 B)^{m-\ell}} \frac{\left|f_{1}\left(y_{1}\right) \cdots f_{m}\left(y_{m}\right)\right|}{\left(\left|x-y_{1}\right|+\cdots+\left|x-y_{m}\right|\right)^{m n-\alpha}} \\
& \times d y_{1} \cdots d y_{m} \\
& \leq C \prod_{i=\ell+1}^{m} \int_{2 B}\left|f_{i}\left(y_{i}\right)\right| d y_{i} \\
& \times \sum_{j=1}^{\infty} \frac{1}{\left|2^{j+1} B\right|^{m-\alpha / n}} \\
& \times \int_{\left(2^{j+1} B\right)^{\ell} \backslash\left(2^{j} B\right)^{\ell}}\left|f_{1}\left(y_{1}\right) \cdots f_{\ell}\left(y_{\ell}\right)\right| d y_{1} \cdots d y_{\ell} \\
& \leq C \prod_{i=\ell+1}^{m} \int_{2 B}\left|f_{i}\left(y_{i}\right)\right| d y_{i} \\
& \times \sum_{j=1}^{\infty} \frac{1}{\left|2^{j+1} B\right|^{m-\alpha / n}} \prod_{i=1}^{\ell} \int_{2^{j+1} B \backslash 2^{j} B}\left|f_{i}\left(y_{i}\right)\right| d y_{i} \\
& \leq C \sum_{j=1}^{\infty} \prod_{i=1}^{m} \frac{1}{\left|2^{j+1} B\right|^{1-\alpha / m n}} \int_{2^{j+1} B}\left|f_{i}\left(y_{i}\right)\right| d y_{i},
\end{aligned}
$$

and we arrive at the expression considered in the previous case. Thus, for any $x \in B$, we also have

$$
\begin{gathered}
\left|I_{\alpha}\left(f_{1}^{\infty}, \ldots, f_{\ell}^{\infty}, f_{\ell+1}^{0}, \ldots, f_{m}^{0}\right)(x)\right| \\
\leq C \prod_{i=1}^{m}\left\|f_{i}\right\|_{L^{p_{i}}, \kappa p_{i} q / p q_{i}\left(w_{i}^{p_{i}}, w_{i}^{q_{i}}\right)} \\
\cdot \sum_{j=1}^{\infty} v_{\vec{w}}^{q}\left(2^{j+1} B\right)^{\kappa / p-1 / q} .
\end{gathered}
$$


Therefore, by the inequality (64) and the above pointwise inequality, we obtain

$$
\begin{aligned}
J^{\alpha_{1}, \ldots, \alpha_{m}} & \leq \nu_{\vec{w}}^{q}(B)^{1 / q-\kappa / p}\left|I_{\alpha}\left(f_{1}^{\infty}, \ldots, f_{\ell}^{\infty}, f_{\ell+1}^{0}, \ldots, f_{m}^{0}\right)(x)\right| \\
\leq & C \prod_{i=1}^{m}\left\|f_{i}\right\|_{L^{p_{i}, \kappa p_{i}} q / p q_{i}\left(w_{i}^{p_{i}}, w_{i}^{q_{i}}\right)} \cdot \sum_{j=1}^{\infty} \frac{v_{\vec{w}}^{q}(B)^{1 / q-\kappa / p}}{\nu_{\vec{w}}^{q}\left(2^{j+1} B\right)^{1 / q-\kappa / p}} \\
\leq & C \prod_{i=1}^{m}\left\|f_{i}\right\|_{L^{p_{i}, \kappa p_{i}} q / p q_{i}\left(w_{i}^{p_{i}}, w_{i}^{q_{i}}\right)} \\
& \cdot \sum_{j=1}^{\infty}\left(\frac{|B|}{\left|2^{j+1} B\right|}\right)^{\delta^{\prime}(1 / q-\kappa / p)} \\
\leq & C \prod_{i=1}^{m}\left\|f_{i}\right\|_{L^{p_{i}, \kappa p_{i}} q / p q_{i}\left(w_{i}^{p_{i}}, w_{i}^{q_{i}}\right)}
\end{aligned}
$$

Summarizing the estimates derived above and then taking the supremum over all balls $B \subseteq \mathbb{R}^{n}$, we finish the proof of Theorem 3.

Proof of Theorem 4. As before, fix a ball $B=B\left(x_{0}, r_{B}\right) \subseteq \mathbb{R}^{n}$ and split $f_{i}$ into $f_{i}=f_{i}^{0}+f_{i}^{\infty}$, where $f_{i}^{0}=f_{i} \chi_{2 B}, i=1, \ldots, m$. Then for each fixed $\lambda>0$, we can write

$$
\begin{aligned}
\nu_{\vec{w}}^{q}\left(\left\{x \in B:\left|I_{\alpha}\left(f_{1}, \ldots, f_{m}\right)\right|>\lambda\right\}\right)^{1 / q} \\
\leq \nu_{\vec{w}}^{q}\left(\left\{x \in B:\left|I_{\alpha}\left(f_{1}^{0}, \ldots, f_{m}^{0}\right)\right|>\frac{\lambda}{2^{m}}\right\}\right)^{1 / q} \\
\quad+\sum^{\prime} \nu_{\vec{w}}^{q}\left(\left\{x \in B:\left|I_{\alpha}\left(f_{1}^{\alpha_{1}}, \ldots, f_{m}^{\alpha_{m}}\right)\right|>\frac{\lambda}{2^{m}}\right\}\right)^{1 / q} \\
=J_{*}^{0}+\sum^{\prime} J_{*}^{\alpha_{1}, \ldots, \alpha_{m}},
\end{aligned}
$$

where each term of $\sum^{\prime}$ contains at least one $\alpha_{i} \neq 0$. By Lemma 9 again, we know that $\left(\nu_{\vec{w}}\right)^{q} \in A_{m q}$ with $1<m q<\infty$. Using Theorem $\mathrm{D}$ and Lemmas 16 and 5, we have

$$
\begin{aligned}
J_{*}^{0} & \leq \frac{C}{\lambda} \prod_{i=1}^{m}\left(\int_{2 B}\left|f_{i}(x)\right|^{p_{i}} w_{i}(x)^{p_{i}} d x\right)^{1 / p_{i}} \\
& \leq \frac{C \cdot \prod_{i=1}^{m} w_{i}^{q_{i}}(2 B)^{\kappa q / p q_{i}}}{\lambda} \prod_{i=1}^{m}\left\|f_{i}\right\|_{L^{p_{i}, \kappa p_{i}} q / p q_{i}}\left(w_{i}^{p_{i}}, w_{i}^{q_{i}}\right) \\
& \leq \frac{C \cdot \nu_{\vec{w}}^{q}(2 B)^{\kappa / p}}{\lambda} \prod_{i=1}^{m}\left\|f_{i}\right\|_{L^{p_{i}}, \kappa p_{i} q / p q_{i}}\left(w_{i}^{p_{i}}, w_{i}^{q_{i}}\right) \\
& \leq \frac{C \cdot \nu_{\vec{w}}^{q}(B)^{\kappa / p}}{\lambda} \prod_{i=1}^{m}\left\|f_{i}\right\|_{L^{p_{i}}, \kappa p_{i} q / p q_{i}}\left(w_{i}^{p_{i}}, w_{i}^{q_{i}}\right) .
\end{aligned}
$$

In the proof of Theorem 3, we have already proved the following pointwise estimate (see (62) and (66)). Consider

$$
\begin{aligned}
& \left|I_{\alpha}\left(f_{1}^{\alpha_{1}}, \ldots, f_{m}^{\alpha_{m}}\right)(x)\right| \\
& \quad \leq C \sum_{j=1}^{\infty} \prod_{i=1}^{m} \frac{1}{\left|2^{j+1} B\right|^{1-\alpha / m n}} \int_{2^{j+1} B}\left|f_{i}\left(y_{i}\right)\right| d y_{i} .
\end{aligned}
$$

Without loss of generality, we may assume that $p_{1}=\cdots=$ $p_{\ell}=\min \left\{p_{1}, \ldots, p_{m}\right\}=1$ and $p_{\ell+1}, \ldots, p_{m}>1$. By using Hölder's inequality, the multiple $A_{\vec{P}, q}$ condition, and Lemma 16, we obtain

$$
\begin{aligned}
\mid I_{\alpha}( & \left.f_{1}^{\alpha_{1}}, \ldots, f_{m}^{\alpha_{m}}\right)(x) \mid \\
\leq & C \sum_{j=1}^{\infty} \prod_{i=1}^{\ell} \frac{1}{\left|2^{j+1} B\right|^{1-\alpha / m n}} \int_{2^{j+1} B}\left|f_{i}\left(y_{i}\right)\right| d y_{i} \\
& \times \prod_{i=\ell+1}^{m} \frac{1}{\left.2^{j+1} B\right|^{1-\alpha / m n}} \int_{2^{j+1} B}\left|f_{i}\left(y_{i}\right)\right| d y_{i} \\
\leq & C \sum_{j=1}^{\infty} \prod_{i=1}^{\ell} \frac{1}{\left|2^{j+1} B\right|^{1-\alpha / m n}} \\
& \times \int_{2^{j+1} B}\left|f_{i}\left(y_{i}\right)\right| w_{i}\left(y_{i}\right) d y_{i}\left(\inf _{y_{i} \in 2^{j+1} B} w_{i}\left(y_{i}\right)\right)^{-1} \\
& \times \prod_{i=\ell+1}^{m} \frac{1}{\left|2^{j+1} B\right|^{1-\alpha / m n}}\left(\int_{2^{j+1} B}\left|f_{i}\left(y_{i}\right)\right|^{p_{i}} w_{i}\left(y_{i}\right)^{p_{i}} d y_{i}\right)^{1 / p_{i}} \\
& \times\left(\int_{2^{j+1} B} w_{i}\left(y_{i}\right)^{-p_{i}^{\prime}} d y_{i}\right)^{1 / p_{i}^{\prime}} \\
\leq & C \prod_{i=1}^{m}\left\|f_{i}\right\|_{L^{p_{i}, \kappa p_{i}} q / p q_{i}\left(w_{i}^{p_{i}}, w_{i}^{q_{i}}\right)} \cdot \sum_{j=1}^{\infty} v_{\vec{w}}^{q}\left(2^{j+1} B\right)^{\kappa / p-1 / q} .
\end{aligned}
$$

Note that $\left(\nu_{\vec{w}}\right)^{q} \in A_{m q}$ with $1<m q<\infty$. Hence, it follows from the inequality (64) that, for any $x \in B$,

$$
\begin{aligned}
& \left|I_{\alpha}\left(f_{1}^{\alpha_{1}}, \ldots, f_{m}^{\alpha_{m}}\right)(x)\right| \\
& =C \prod_{i=1}^{m}\left\|f_{i}\right\|_{L^{p_{i}}, \kappa p_{i} q / p q_{i}}\left(w_{i}^{p_{i}}, w_{i}^{q_{i}}\right) \\
& \cdot \frac{1}{\nu_{\vec{w}}^{q}(B)^{1 / q-\kappa / p}} \sum_{j=1}^{\infty} \frac{\nu_{\vec{w}}^{q}(B)^{1 / q-\kappa / p}}{\nu_{\vec{w}}^{q}\left(2^{j+1} B\right)^{1 / q-\kappa / p}} \\
& \leq C \prod_{i=1}^{m}\left\|f_{i}\right\|_{L^{p_{i}, \kappa p_{i}} q / p q_{i}}\left(w_{i}^{p_{i}}, w_{i}^{q_{i}}\right) \\
& \cdot \frac{1}{v_{\vec{w}}^{q}(B)^{1 / q-\kappa / p}} \sum_{j=1}^{\infty}\left(\frac{|B|}{\left|2^{j+1} B\right|}\right)^{\delta^{\prime}(1 / q-\kappa / p)} \\
& \leq C \prod_{i=1}^{m}\left\|f_{i}\right\|_{L^{p_{i}, \kappa p_{i} q / p q_{i}}\left(w_{i}^{p_{i}}, w_{i}^{q_{i}}\right)} \cdot \frac{1}{\nu_{\vec{w}}^{q}(B)^{1 / q-\kappa / p}} .
\end{aligned}
$$

If $\left\{x \in B:\left|I_{\alpha}\left(f_{1}^{\alpha_{1}}, \ldots, f_{m}^{\alpha_{m}}\right)(x)\right|>\lambda / 2^{m}\right\}=\varnothing$, then the inequality

$$
J_{*}^{\alpha_{1}, \ldots, \alpha_{m}} \leq \frac{C \cdot \nu_{\vec{w}}^{q}(B)^{\kappa / p}}{\lambda} \prod_{i=1}^{m}\left\|f_{i}\right\|_{L^{p_{i}, \kappa p_{i}} q / p q_{i}}\left(w_{i}^{p_{i}}, w_{i}^{q_{i}}\right)
$$

holds trivially. Now, if instead we assume that $\{x \in B$ : $\left.\left|I_{\alpha}\left(f_{1}^{\alpha_{1}}, \ldots, f_{m}^{\alpha_{m}}\right)(x)\right|>\lambda / 2^{m}\right\} \neq \varnothing$, then, by the pointwise inequality (73), we get

$$
\lambda<C \prod_{i=1}^{m}\left\|f_{i}\right\|_{L^{p_{i}, \kappa \kappa_{i} q / p q_{i}}\left(w_{i}^{p_{i}}, w_{i}^{q_{i}}\right)} \cdot \frac{1}{\nu_{\vec{w}}^{q}(B)^{1 / q-\kappa / p}},
$$


which in turn gives that

$$
\nu_{\vec{w}}^{q}(B)^{1 / q} \leq \frac{C \cdot v_{\vec{w}}^{q}(B)^{\kappa / p}}{\lambda} \prod_{i=1}^{m}\left\|f_{i}\right\|_{L^{p_{i}, \kappa p_{i} q / p q_{i}}\left(w_{i}^{p_{i}}, w_{i}^{q_{i}}\right)}
$$

Therefore,

$$
J_{*}^{\alpha_{1}, \ldots, \alpha_{m}} \leq \nu_{\vec{w}}^{q}(B)^{1 / q} \leq \frac{C \cdot \nu_{\vec{w}}^{q}(B)^{\kappa / p}}{\lambda} \prod_{i=1}^{m}\left\|f_{i}\right\|_{L^{p_{i}, \kappa p_{i} q / p q_{i}}\left(w_{i}^{p_{i}}, w_{i}^{q_{i}}\right)} .
$$

Collecting all the above estimates and then taking the supremum over all balls $B \subseteq \mathbb{R}^{n}$ and all $\lambda>0$, we conclude the proof of Theorem 4 .

By using Hölder's inequality, it is easy to verify that if $1 \leq$ $p_{i}<q_{i}, 1 / q=\sum_{k=1}^{m} 1 / q_{k}$, and each $w_{i}$ is in $A_{p_{i}, q_{i}}$, then we have

$$
\prod_{i=1}^{m} A_{p_{i}, q_{i}} \subset A_{\vec{P}, q}
$$

and this inclusion is strict (see [17]). Also, recall that $w \in A_{p, q}$ if and only if $w^{q} \in A_{1+q / p^{\prime}} \subset A_{\infty}$ (see [36]). Thus, as straightforward consequences of Theorems 3 and 4 , we finally obtain the following.

Corollary 17. Let $m \geq 2$, let $0<\alpha<m n$, and let $I_{\alpha}$ be an $m$-linear fractional integral operator. If $p_{1}, \ldots, p_{m} \in(1, \infty)$, $1 / p=\sum_{k=1}^{m} 1 / p_{k}, 1 / q_{k}=1 / p_{k}-\alpha / m n$ and $1 / q=\sum_{k=1}^{m} 1 / q_{k}=$ $1 / p-\alpha / n$, and $\vec{w}=\left(w_{1}, \ldots, w_{m}\right) \in \prod_{i=1}^{m} A_{p_{i}, q_{i}}$, then, for any $0<\kappa<p / q$, there exists a constant $C>0$ independent of $\vec{f}=\left(f_{1}, \ldots, f_{m}\right)$ such that

$$
\left\|I_{\alpha}(\vec{f})\right\|_{L^{q, \kappa q / p}\left(\left(v_{\vec{w}}\right)^{q}\right)} \leq C \prod_{i=1}^{m}\left\|f_{i}\right\|_{L^{p_{i}, \kappa p_{i} q / p q_{i}}\left(w_{i}^{p_{i}}, w_{i}^{q_{i}}\right)},
$$

where $v_{\vec{w}}=\prod_{i=1}^{m} w_{i}$.

Corollary 18. Let $m \geq 2$, let $0<\alpha<m n$, and let $I_{\alpha}$ be an $m$-linear fractional integral operator. If $p_{1}, \ldots, p_{m} \in[1, \infty)$, $\min \left\{p_{1}, \ldots, p_{m}\right\}=1,1 / p=\sum_{k=1}^{m} 1 / p_{k}, 1 / q_{k}=1 / p_{k}-\alpha / m n$ and $1 / q=\sum_{k=1}^{m} 1 / q_{k}=1 / p-\alpha / n$, and $\vec{w}=\left(w_{1}, \ldots, w_{m}\right) \epsilon$ $\prod_{i=1}^{m} A_{p_{i}, q_{i}}$, then, for any $0<\kappa<p / q$, there exists a constant $C>0$ independent of $\vec{f}=\left(f_{1}, \ldots, f_{m}\right)$ such that

$$
\left\|I_{\alpha}(\vec{f})\right\|_{W L^{q, \kappa q / p}\left(\left(v_{\vec{w}}\right)^{q}\right)} \leq C \prod_{i=1}^{m}\left\|f_{i}\right\|_{L^{p_{i}, \kappa p_{i} q / p q_{i}}\left(w_{i}^{p_{i}}, w_{i}^{q_{i}}\right)}
$$

where $v_{\vec{w}}=\prod_{i=1}^{m} w_{i}$.

\section{References}

[1] R. R. Coifman and Y. Meyer, "On commutators of singular integrals and bilinear singular integrals," Transactions of the American Mathematical Society, vol. 212, pp. 315-331, 1975.

[2] L. Grafakos and R. H. Torres, "Multilinear Calderón-Zygmund theory," Advances in Mathematics, vol. 165, no. 1, pp. 124-164, 2002.
[3] L. Grafakos and R. H. Torres, "Maximal operator and weighted norm inequalities for multilinear singular integrals," Indiana University Mathematics Journal, vol. 51, no. 5, pp. 1261-1276, 2002.

[4] L. Grafakos and R. H. Torres, "On multilinear singular integrals of Calderón-Zygmund type," Publicacions Matemàtiques, pp. 57-91, 2002.

[5] L. Grafakos and N. Kalton, "Multilinear Calderón-Zygmund operators on Hardy spaces," Collectanea Mathematica, vol. 52, no. 2, pp. 169-179, 2001.

[6] G. E. Hu and Y. Meng, "Multilinear Calderón-Zygmund operator on products of Hardy spaces," Acta Mathematica Sinica (English Series), vol. 28, no. 2, pp. 281-294, 2012.

[7] W. J. Li, Q. Y. Xue, and K. Yabuta, "Maximal operator for multilinear Calderón-Zygmund singular integral operators on weighted Hardy spaces," Journal of Mathematical Analysis and Applications, vol. 373, no. 2, pp. 384-392, 2011.

[8] W. J. Li, Q. Y. Xue, and K. Yabuta, "Multilinear CalderónZygmund operators on weighted Hardy spaces," Studia Mathematica, vol. 199, no. 1, pp. 1-16, 2010.

[9] A. K. Lerner, S. Ombrosi, C. Pérez, R. H. Torres, and R. TrujilloGonzález, "New maximal functions and multiple weights for the multilinear Calderón-Zygmund theory," Advances in Mathematics, vol. 220, no. 4, pp. 1222-1264, 2009.

[10] L. Grafakos, “On multilinear fractional integrals," Studia Mathematica, vol. 102, no. 1, pp. 49-56, 1992.

[11] T. Iida, E. Sato, Y. Sawano, and H. Tanaka, "Weighted norm inequalities for multilinear fractional operators on Morrey spaces," Studia Mathematica, vol. 205, no. 2, pp. 139-170, 2011.

[12] T. Iida, E. Sato, Y. Sawano, and H. Tanaka, "Multilinear fractional integrals on Morrey spaces," Acta Mathematica Sinica (English Series), vol. 28, no. 7, pp. 1375-1384, 2012.

[13] T. Iida, E. Sato, Y. Sawano, and H. Tanaka, "Sharp bounds for multilinear fractional integral operators on Morrey type spaces," Positivity, vol. 16, no. 2, pp. 339-358, 2012.

[14] C. E. Kenig and E. M. Stein, "Multilinear estimates and fractional integration," Mathematical Research Letters, vol. 6, no. 1, pp. 1-15, 1999.

[15] G. Pradolini, "Weighted inequalities and pointwise estimates for the multilinear fractional integral and maximal operators," Journal of Mathematical Analysis and Applications, vol. 367, no. 2, pp. 640-656, 2010.

[16] L. Tang, "Endpoint estimates for multilinear fractional integrals," Journal of the Australian Mathematical Society, vol. 84, no. 3, pp. 419-429, 2008.

[17] K. Moen, "Weighted inequalities for multilinear fractional integral operators," Collectanea Mathematica, vol. 60, no. 2, pp. 213-238, 2009.

[18] X. Chen and Q. Y. Xue, "Weighted estimates for a class of multilinear fractional type operators," Journal of Mathematical Analysis and Applications, vol. 362, no. 2, pp. 355-373, 2010.

[19] C. B. Morrey, "On the solutions of quasi-linear elliptic partial differential equations," Transactions of the American Mathematical Society, vol. 43, no. 1, pp. 126-166, 1938.

[20] D. R. Adams, "A note on Riesz potentials," Duke Mathematical Journal, vol. 42, no. 4, pp. 765-778, 1975.

[21] F. Chiarenza and M. Frasca, "Morrey spaces and HardyLittlewood maximal function," Rendiconti di Matematica e delle sue Applicazioni, vol. 7, no. 3-4, pp. 273-279, 1987.

[22] J. Peetre, “On the theory of $\mathscr{L}_{p, \lambda}$ spaces," Journal of Functional Analysis, vol. 4, pp. 71-87, 1969. 
[23] D. S. Fan, S. Z. Lu, and D. C. Yang, "Regularity in Morrey spaces of strong solutions to nondivergence elliptic equations with VMO coefficients," Georgian Mathematical Journal, vol. 5, no. 5, pp. 425-440, 1998.

[24] G. Di Fazio and M. A. Ragusa, "Interior estimates in Morrey spaces for strong solutions to nondivergence form equations with discontinuous coefficients," Journal of Functional Analysis, vol. 112, no. 2, pp. 241-256, 1993.

[25] G. Di Fazio, D. K. Palagachev, and M. A. Ragusa, "Global Morrey regularity of strong solutions to the Dirichlet problem for elliptic equations with discontinuous coefficients," Journal of Functional Analysis, vol. 166, no. 2, pp. 179-196, 1999.

[26] Y. Komori and S. Shirai, "Weighted Morrey spaces and a singular integral operator," Mathematische Nachrichten, vol. 282, no. 2, pp. 219-231, 2009.

[27] H. Wang, "Some estimates for commutators of CalderónZygmund operators on the weighted Morrey spaces," Scientia Sinica Mathematica, vol. 42, no. 1, pp. 31-45, 2012.

[28] H. Wang, "The boundedness of some operators with rough kernel on the weighted Morrey spaces," Acta Mathematica Sinica (Chinese Series), vol. 55, no. 4, pp. 589-600, 2012.

[29] H. Wang, "Intrinsic square functions on the weighted Morrey spaces," Journal of Mathematical Analysis and Applications, vol. 396, no. 1, pp. 302-314, 2012.

[30] H. Wang, "Boundedness of fractional integral operators with rough kernels on weighted Morrey spaces," Acta Mathematica Sinica (Chinese Series), vol. 56, pp. 175-186, 2013.

[31] H. Wang, "Some estimates for commutators of fractional integral operators on weighted Morrey spaces," Acta Mathematica Sinica (Chinese Series), vol. 56, pp. 889-906, 2013.

[32] H. Wang, "Some estimates for commutators of fractional integrals associated to operators with Gaussian kernel bounds on weighted Morrey spaces," Analysis in Theory and Applications, vol. 29, pp. 72-85, 2013.

[33] H. Wang, "Weak type estimates for intrinsic square functions on weighted Morrey spaces," Analysis in Theory and Applications, vol. 29, pp. 104-119, 2013.

[34] H. Wang and H. P. Liu, "Some estimates for Bochner-Riesz operators on the weighted Morrey spaces," Acta Mathematica Sinica (Chinese Series), vol. 55, no. 3, pp. 551-560, 2012.

[35] B. Muckenhoupt, "Weighted norm inequalities for the Hardy maximal function," Transactions of the American Mathematical Society, vol. 165, pp. 207-226, 1972.

[36] B. Muckenhoupt and R. Wheeden, "Weighted norm inequalities for fractional integrals," Transactions of the American Mathematical Society, vol. 192, pp. 261-274, 1974.

[37] J. García-Cuerva and J. L. Rubio de Francia, Weighted Norm Inequalities and Related Topics, vol. 116 of North-Holland Mathematics Studies, North-Holland Publishing, Amsterdam, The Netherlands, 1985.

[38] J. Duoandikoetxea, Fourier Analysis, vol. 29 of Graduate Studies in Mathematics, American Mathematical Society, Providence, RI, USA, 2000. 


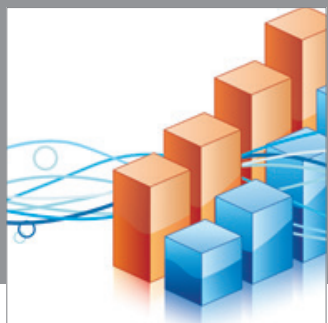

Advances in

Operations Research

mansans

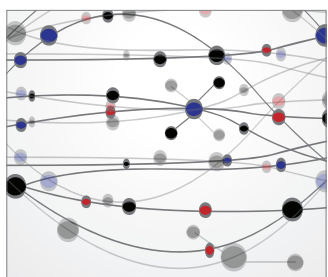

The Scientific World Journal
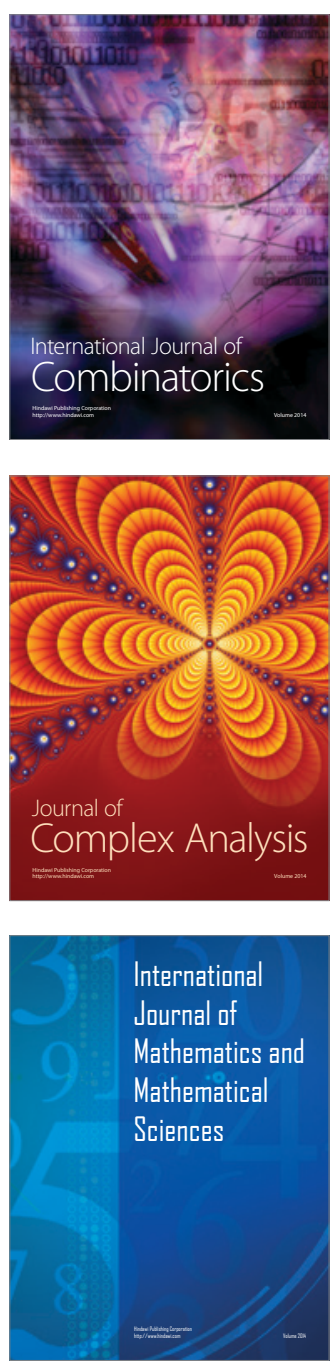
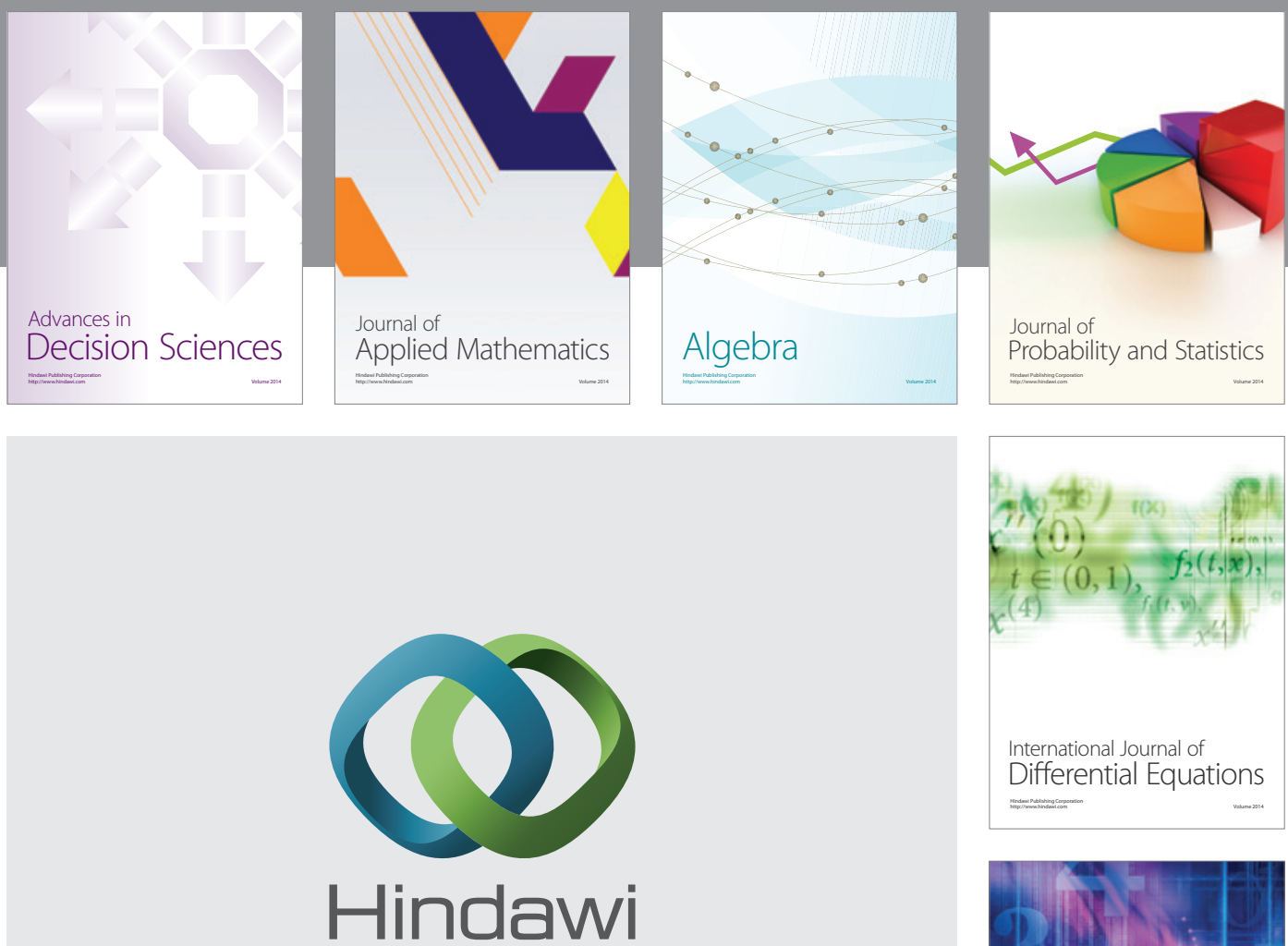

Submit your manuscripts at http://www.hindawi.com
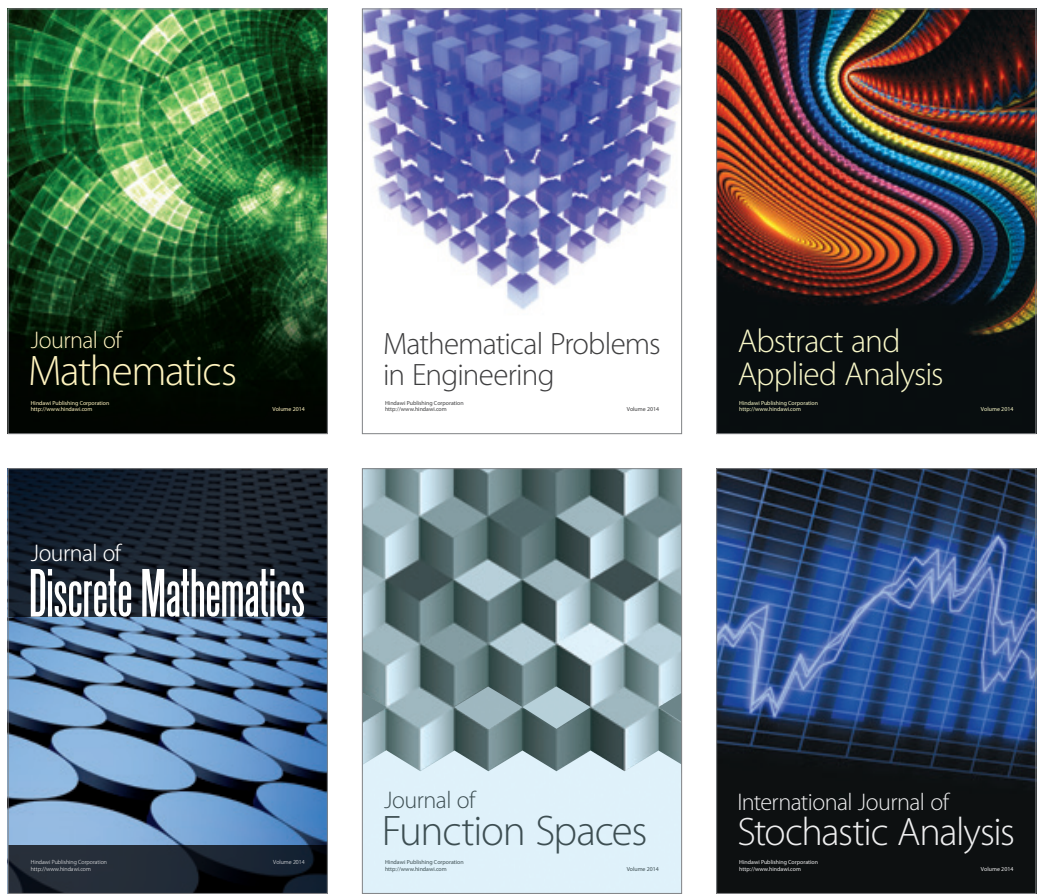

Journal of

Function Spaces

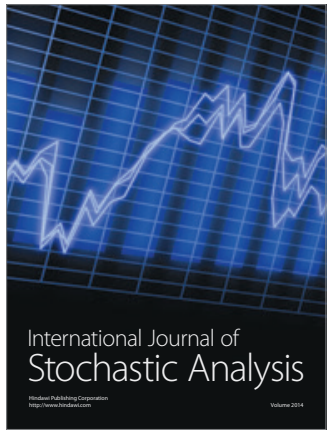

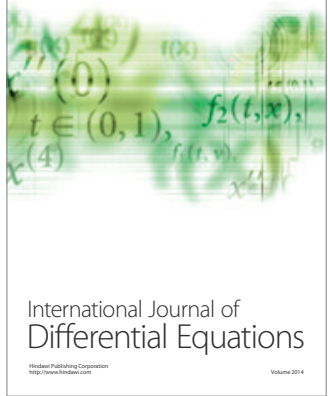
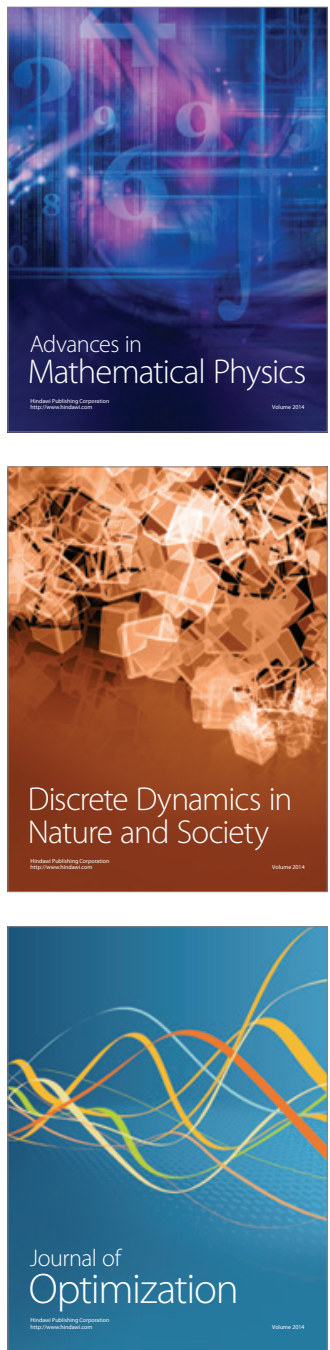